\title{
ON CONTINUITY OF INVERSE OPERATORS
}

\author{
BY W. SEOWIKOWSKI ${ }^{1}$ \\ Communicated by Einar Hille, March 1, 1961
}

This paper concerns the so-called "closed graph theorem" or "open mapping theorem" (cf. $[2 ; 3 ; 5 ; 6])$. We intend to introduce here only the main result. Discussion is restricted to some general indications as to applications of the theorem proved. The full paper will be published in Studia Mathematica.

Suppose we have been given countable sets $T, S$, a subset $N$ of the set $T^{S}$ of all $T$-valued functions defined on $S$ and a family of linear spaces with pseudonorms ${ }^{2} X_{p, q},|\cdot|_{p, q}$, where $(p, q)$ runs over the union of all graphs from $N$ and all $X_{p, q}$ are linear subspaces of the same linear space $X$. Denote briefly $\left(N, X_{p, q},|\cdot|_{p, q}\right)$ by $\mathfrak{F}$. Having fixed $\mathfrak{F}$ we associate with each $\left(p_{q}\right) \in N$ a linear space with pseudonorm defined as follows:

$$
X_{\left(p_{q}\right)}=\bigcap_{q \in S} X_{p_{q}, q} ; \quad|x|_{\left(p_{q}\right)}=\sum_{n=1}^{\infty} 2^{-n}|x|_{p_{q_{n}}, q_{n}}\left(1+|x|_{p_{q_{n}}, q_{n}}\right)^{-1},
$$

where $S=q_{1}, q_{2}, \cdots$. Assuming $X=\bigcup_{\left(p_{q}\right) \in N} X_{\left(p_{q}\right)}$ we introduce $\mathfrak{F}$ boundedness in $X$ as follows: a sequence $\left(x_{n}\right) \subset X$ is said to be $\mathfrak{F}-$ bounded if $\left(x_{n}\right)$ is bounded in at least one $X_{\left(p_{q}\right)},|\cdot|_{\left(p_{q}\right)}$ in the usual sense. Suppose there is given a BC topology $(\tau)$ in $X .^{3}$ The topology $(\tau)$ is said to be represented by $\mathfrak{F}$ if the notions of $\mathfrak{F}$-boundedness and $(\tau)$-boundedness coincide.

If for given $\mathfrak{F}$ there exists at least one $\mathrm{BC}$ topology that is represented by $\mathfrak{F}$, then this topology must be given by considering a pseudonorm being continuous on $X$ iff it is continuous restricted to any $X_{\left(p_{q}\right)},|\cdot|_{\left(p_{q}\right)}$, with $\left(p_{q}\right) \in N$. Therefore there is at most one BC topology that is represented by given $F$ and we denote this topology by $\left(\tau_{\mathfrak{F}}\right)$.

A locally convex topology $(\tau)$ is said to be $(b)$-complete if each bounded mapping of an $h$-normed ${ }^{4}$ space $Z$ into $X$ can be extended to the completion of $Z$. (b)-completeness follows from sequential completeness, and remains after bornologic fortification of the initial topology.

\footnotetext{
${ }^{1}$ Supported by National Science Foundation Grant G 14600.

2 Pseudonorms are not necessarily homogeneous.

${ }^{3} \mathrm{BC}=$ bornologic locally convex.

4 $h$-norm $=$ homogenous norm.
} 
Two topologies $\left(\tau_{1}\right)$ and $\left(\tau_{2}\right)$ are said to be $\sigma$-compatible if from the fact that $\left(x_{n}\right)$ tends to $x_{1}$ in $\left(\tau_{1}\right)$ topology and to $x_{2}$ in $\left(\tau_{2}\right)$ topology follows that $x_{1}=x_{2}$.

The purpose of this paper is to prove that under some conditions listed below as $(\alpha),(\beta)$ and $(\gamma)$ and concerning $\mathfrak{F}$, each $(b)$-complete BC topology of $X$ which is $\sigma$-comparable with $\left(\tau_{\mathcal{F}}\right)$ is identical with $\left(\tau_{\mathcal{F}}\right)$, provided that $\left(\tau_{\mathcal{F}}\right)$ is represented by $\mathfrak{F}$ and is itself $(b)$-complete.

Let $N\left(U,\left(p_{q}\right)\right)=\left\{\left(r_{q}\right) \in N: r_{q}=p_{q}\right.$ for $\left.q \in U\right\}$. $\mathfrak{F}$ is said to be $\alpha \beta \gamma$ representation if the following conditions are satisfied:

$(\alpha)$ Given $\left(p_{q}\right) \in T^{s}$. If $N\left(U,\left(p_{q}\right)\right)$ is nonvoid for each finite $U \subset S$ then $\left(p_{q}\right) \in N$.

$(\beta)$ Given $\left(p_{q}\right) \in N$ and finite nonvoid $U \subset S$. Then

$$
\bigcap_{q \in U} X_{p_{q}, q} \subset \underset{\left(k_{q}\right) \in N\left(U,\left(p_{q}\right)\right.}{\bigcup} X_{k_{r}, r} \text { and } \underset{\left(k_{q}\right) \in N}{\bigcup} X_{k_{r}, r}=X \text { for each } r \in S \text {. }
$$

$(\gamma)$ Given $S=\cup_{n=1}^{\infty} U_{n}$, where $U_{n} \subset U_{n+1}$-finite nonvoid, $\left(p_{q}\right) \in N$ and

$$
x_{n} \in \bigcap_{q \in U_{n}} X_{p_{q}, q}, \quad \lim _{n} \sup _{\boldsymbol{k}}\left|\sum_{i=1}^{k} x_{n+i}\right|_{p_{q}, q}=0 \quad \text { for } q \in S .
$$

Then there exists $\left(p_{q}^{*}\right) \in N\left(U_{1},\left(p_{q}\right)\right)$ such that $\left(x_{n}\right) \subset X_{\left(p_{q}^{*}\right)}$ and

$$
\lim _{\boldsymbol{n}} \sup _{\boldsymbol{k}}\left|\sum_{i=1}^{k} x_{n+i}\right|\left(\boldsymbol{p}^{*}\right)=0 \text {. }
$$

Conditions $(\alpha),(\beta)$ and $(\gamma)$ are set in such a way that starting with $\alpha \beta \gamma$-representable spaces and constructing spaces of linear transformations, dual spaces, tensorial products, countable projective and inductive limits, we have the natural $\alpha \beta \gamma$-representations for the new spaces.

In order to follow the main theorem we will concentrate on two very important examples.

Let $D$ be the space of all infinitely differentiable functions with compact carriers defined on an open convex subset $\Omega$ of $k$-dimensional Euclidean space and suppose $\Omega$ is the union of compact convex subsets $\Omega_{p}$, where $\Omega_{p} \subset$ Int $\Omega_{p+1}$. We set for $\phi \in D$

$$
|\phi|_{p, q}=\sup \left\{\left|D^{r} \phi(\lambda)\right|: \lambda \in \Omega_{p},|r|<q\right\},
$$

where $r=\left(r_{1}, \cdots, r_{k}\right),|r|=r_{1}+\cdots+r_{k}$ and all $p, q, r_{i}$ are positive integers. We set further

$$
D_{p, q}=\left\{\phi \in D:|\phi|_{p, q}<\infty, \phi \text { vanishes outside } \Omega_{p}\right\} .
$$

The $\left(\tau_{\xi}\right)$ topology induced by $\mathfrak{F}=\left(T^{S}, D_{p, q},|\cdot|_{p, q}\right)$ is the initial topology of $D$ as introduced in [4] and is $\alpha \beta \gamma$-represented by $\mathfrak{F}$. 
Let now $\mathscr{D}^{\prime}$ be the dual of $\mathscr{D}$, i.e. the space of all distributions over $\Omega$. We set for $T \in \mathbb{D}^{\prime}$

$$
\begin{aligned}
\|T\|_{p, q} & =\sup \left\{|T \phi|:|\phi|_{q, p} \leqq 1 ; \phi \in D_{q, p}\right\}, \\
\mathscr{D}_{p, q}^{\prime} & =\left\{T \in \mathscr{D}^{\prime}:\|T\|_{p, q}<\infty\right\} .
\end{aligned}
$$

Again $\left(\tau \mathscr{F}^{\prime}\right)$ is $\alpha \beta \gamma$-represented by $\mathfrak{F}^{\prime}=\left(T^{S}, \mathscr{D}_{p, q}^{\prime},\|\cdot\|_{p, q}\right)$ and coincide with the strong topology of $\mathfrak{D}^{\prime}$.

Theorem. (A) Suppose $\mathfrak{F}=\left(N, X_{p, q},|\cdot|_{p, q}\right)$ satisfies $(\alpha)(\beta)$ and $(\gamma), X=U_{\left(p_{q}\right) \in N} X_{\left(p_{q}\right)}$, all spaces $X_{\left(p_{q}\right)},|\cdot|_{\left(p_{q}\right)}$ are complete, $Z,\|\cdot\|$ is a complete normed space, $Z$ is a linear subset of $X$ and if $\left(x_{n}\right) \subset X_{\left(p_{q}\right)} \cap Z$, $\left\|x_{n}-x_{1}\right\| \rightarrow 0,\left|x_{n}-x_{2}\right|_{\left(p_{q}\right)} \rightarrow 0$, then $x_{1}=x_{2}$.

Then there exists $\left(p_{q}\right) \in N$ such that $Z \subset X_{\left(p_{q}\right)}$ and for $\left(x_{n}\right) \subset Z\left\|x_{n}\right\| \rightarrow 0$ implies $\left|x_{n}\right|_{\left(p_{q}\right)} \rightarrow 0$.

(B) Suppose that there exists an $\alpha \beta \gamma$-representation of a (b)-complete $\mathrm{BC}$ space $X,(\tau)$. Then, whatever is an $\alpha \beta \gamma(b)$-complete $\mathrm{BC}$ topology $(\rho)$ in $X,(\rho)$ is the same as $(\tau)$ provided $(\rho)$ and $(\tau)$ are compatible.

It is significant that even in the case of distribution spaces the theorem yields the not trivial fact that all one-to-one continuous mappings of $D^{\prime}$ onto $D^{\prime}$ have the continuous inverses.

PROOF OF THE THEOREM. Suppose we have proved the following lemmas:

Lemma 1. From $(\alpha)$ and $(\beta)$ follows that there exists $\left(p_{q}\right) \in N$ such that for each finite nonvoid $U \subset S$ the set $Z \cap\left(\cap_{q \in U} X_{p_{q}, q}\right)$ is of the second category in $Z$.

LEMMA 2 (BANACH). Let us consider two normed linear spaces $W,\|\cdot\|$ and $V,|\cdot|$ and let $V$ be a second category linear subset of $W,\|\cdot\|$. Then to each $\epsilon>0$ there corresponds $\eta>0$ such that

$$
[\{x \in V:|x|<\epsilon\}]^{-} \supset\{y \in W:\|y\|<\eta\},
$$

where the closure "-" is taken in $W,\|\cdot\|$.

To prove the theorem we take $\left(p_{q}\right)$ from Lemma 1, arbitrary $\epsilon>0$ and a finite nonvoid $U \subset S$. Suppose $\left(q_{1}, q_{2}, \cdots\right)$ is a sequence of all elements from $S-U$. We set $U_{0}=U$ and $U_{i}=U_{i-1} \cup\left\{q_{i}\right\}$ for $i \geqq 1$. Denote $Z_{n}=\left(\bigcap_{q \in U_{n}} X_{p_{q}, q}\right) \cap Z$. From Lemma 2 follows that

$$
\left\{y \in Z:\|y\|<\eta_{n}\right\} \subset\left[\left\{x \in Z_{n-1}:|x|_{n-1}<(1 / 2)^{n} \epsilon\right\}\right]^{-}
$$

holds for some $\left(\eta_{n}\right), n=1,2, \cdots$, where $|x|_{n}=\max \left\{|x|_{p_{q}, q}: q \in U_{n}\right\}$. If $\|y\|<\eta_{1}$, then there exists $x_{1} \in Z_{0}$ with $\left|x_{1}\right|_{0}<(1 / 2) \epsilon$, such that $\left\|y-x_{1}\right\|<\eta_{1}$. Suppose we have $x_{1}, \cdots, x_{n} \in Z$ such that

$\left.{ }^{*}\right) x_{n} \in Z_{n-1},\left|x_{n}\right|_{n-1}<(1 / 2)^{n} \epsilon,\left\|y-\left(x_{1}+\cdots+x_{n}\right)\right\|<\min \left(\eta_{n}, 1 / n\right)$. 
Since $\left\|y-\left(x_{1}+\cdots+x_{n}\right)\right\|<\eta_{n}$, there is $x_{n+1} \in Z_{n}$ with $\left|x_{n+1}\right|_{n}$ $<(1 / 2)^{n+1} \epsilon$ and such that $\left\|\left[y-\left(x_{1}+\cdots+x_{n}\right)\right]-x_{n+1}\right\|$ $<\min \left(\eta_{n+1}, 1 /(n+1)\right)$. Hence there is a sequence $\left(x_{n}\right)$ satisfying $\left(^{*}\right)$ for each $n$. We have for $q \in U_{n}$ and $n \leqq m$

$$
\sup _{k}\left|\sum_{i=m+1}^{m+k} x_{i}\right|_{p_{q}, q} \leqq \sum_{i=m+1}^{\infty}\left|x_{i}\right|_{n} \leqq \sum_{i=m+1}^{\infty}\left|x_{i}\right|_{i-1} \leqq\left(\sum_{i=m+1}^{\infty}(1 / 2)^{i} \epsilon\right)_{m} 0
$$

and thus by $(\gamma),\left(x_{n}\right) \subset X_{\left(p_{q}{ }^{*}\right)}$ and $\sup _{k}\left|\sum_{i=m+1}^{m+k} x_{i}\right|{ }_{\left(p_{q}{ }^{*}\right)} \rightarrow 0$ for some $\left(p_{q}^{*}\right) \in N\left(U,\left(p_{q}\right)\right)$. But $\left\|y-\sum_{i=1}^{m} x_{i}\right\| \rightarrow 0$ and then by the assumption $\left|y-\sum_{i=1}^{m} x_{i}\right|_{\left(p_{q}{ }^{*}\right) \rightarrow 0}$ and hence for $q \in U$ we have $|y|_{p_{q}, q}<\epsilon$. Thus we have proved the following:

If $\|y\|<\eta_{1}$, then $y \in \bigcap_{q \in U} X_{p_{q}, q}$ and $|y|_{p_{q}, q}<\epsilon$ for $q \in U$. Since for each $y \in Z$ there is $m$ such that $\|(1 / m) y\|<\eta_{1}$, we have $y / m \in \bigcap_{q \in U} X_{p_{q}, q}$ and thus $Z \subset \cap_{q \in U} X_{p_{q}, q}$. But $\left(p_{q}\right)$ does not depend on $U$ and thus $Z \subset X_{\left(p_{q}\right)}$ and (A) follows. (B) is just a simple consequence of (A).

Lemma 2 is proved in [1] and here is the proof of Lemma 1. Suppose $S=\left(q_{1}, q_{2}, \cdots\right)$ and let $U_{n}=\left(q_{1}, \cdots, q_{n}\right)$. Since $Z \subset \bigcup_{\left(p_{q}\right) \in N} X_{p_{q_{1}}, q_{1}}$, there is some $\left(p_{q}^{1}\right) \in N$ such that $Z \cap X_{p_{q_{1}}{ }^{1}, q_{1}}$ is of the second category in $Z,\|\cdot\|$. Now, let $\left(p_{q}^{i}\right) \in N, i=1,2, \cdots, n$ be such that $p_{q_{i}}^{k}=p_{q_{i}}^{m}$ for $i \leqq k \leqq m \leqq n$ and let $Z \cap\left(\bigcap_{q \in U_{n}} X_{p_{q}{ }^{n}, q}\right)$ be of the second category in $Z$. By $(\beta)$ we have $\bigcap_{q \in U_{n}} X_{p_{q}{ }^{n}, q} \subset \bigcup_{\left(p_{q}\right) \in N\left(U_{n},\left(p_{q}{ }^{n}\right)\right)} X_{p_{q_{n+1}}, q_{n+1}}$ and then for some $\left(p_{q}^{n+1}\right) \in N\left(U_{n},\left(p_{q}^{n}\right)\right)$ the set

$$
Z \cap X_{p_{q_{n+1}}{ }^{n+1}, q_{n+1}} \cap\left(\bigcap_{q \in U_{n}} X_{p_{q}{ }^{n}, q}\right)
$$

is of the second category in $Z \cap\left(\bigcap_{q \in U_{n}} X_{p_{q}{ }^{n}, q}\right)$. The latter set is linear and therefore dense in $Z$. Hence the former is of the second category in $Z$. Setting $p_{q}=p_{q}{ }^{n}$ for $q=q_{n}$ we have $N\left(U,\left(p_{q}\right)\right)$ nonvoid for each finite $U \subset S$ and then by $(\alpha)$ we have $\left(p_{q}\right) \in N$, which finishes the proof of Lemma 1.

\section{REFERENCES}

1. S. Banach, Théorie des opérations linéaires, Warszawa, Monografje Matematyczne, 1932.

2. V. Pták, Completeness and the open mapping theorem, Bull. Soc. Math. France vol. 86 (1958) pp. 40-74.

3. J. L. Kelly, Hypercomplete linear spaces, Michigan Math. J. vol. 5 (1958) pp. 235-246.

4. L. Schwartz, Theorie des distributions, tomes I, II, Paris, Hermann, 1951, 1957.

5. K. Zeller, Über Stetigkeit von Integraltransformationen, Math. Z. vol. 55 (19511952) pp. 167-182.

6. W. Słowikowski, $(h F)$-spaces and the Banach inversion property, Bull. Acad. Polon. Sci. Cl. III vol. 5 (1957) pp. 487-489.

The Institute for Advanced Study 